\title{
Linear and nonlinear magnetic error measurements using action and phase jump analysis
}

\author{
Javier F. Cardona* \\ National University of Colombia, Ciudad Universitaria, Bogotá, Colombia
}

Stephen G. Peggs

Brookhaven National Laboratory, Upton, New York 11973, USA

(Received 5 August 2008; published 21 January 2009)

\begin{abstract}
"Action and phase jump" analysis is presented - a beam based method that uses amplitude and phase knowledge of a particle trajectory to locate and measure magnetic errors in an accelerator lattice. The expected performance of the method is first tested using single-particle simulations in the optical lattice of the Relativistic Heavy Ion Collider (RHIC). Such simulations predict that under ideal conditions typical quadrupole errors can be estimated within an uncertainty of $0.04 \%$. Other simulations suggest that sextupole errors can be estimated within a 3\% uncertainty. Then the action and phase jump analysis is applied to real RHIC orbits with known quadrupole errors, and to real Super Proton Synchrotron (SPS) orbits with known sextupole errors. It is possible to estimate the strength of a skew quadrupole error from measured RHIC orbits within a $1.2 \%$ uncertainty, and to estimate the strength of a strong sextupole component from the measured SPS orbits within a $7 \%$ uncertainty.
\end{abstract}

DOI: 10.1103/PhysRevSTAB.12.014002

PACS numbers: 41.85.-p, 29.85.Fj, 29.20.-c

\section{INTRODUCTION}

One of the most common methods to measure magnetic errors in high energy accelerators is the matrix response method [1], in which dipole correctors in the accelerator are systematically perturbed while orbit measurements are taken. The optical model of the accelerator-with incompletely known variables like quadrupole strengths, beam position monitors (BPMs) gains, and steering magnet kicks, et cetera-is then fitted to all these measurements. This method has been used successfully in accelerators like the National Synchrotron Light Source and the Advanced Light Source [2] where the number of variables is around 400. Rings as big as the Relativistic Heavy Ion Collider (RHIC) have a significantly larger number of variables (about four thousand), and huge matrices. Procedures to deal with the problems of such large matrices have been used in accelerators like PEP-II, but they are time and resource consuming $[3,4]$.

For these reasons, the application of the matrix response method was deprecated during early RHIC commissioning, in favor of other options like the orbit bump method [5-8], the action phase jump method [9], and others [10-12].

The action and phase jump method considers any optical error-whether a dipole, quadrupole, or sextupole, et cetera-as a simple kick. From this perspective, a particle trajectory is exactly described by two separate equations: one to describe the trajectory just before the error, and one to describe it just after. In both equations the lattice functions assume their original design values, and

\footnotetext{
*jfcardona@unal.edu.co
}

only the action and phase constants differ. The differences between these constants before and after the error allow the strength of the error and its nature to be calculated.

The first advantage of this method is that it is not necessary to generate new optics including the effect of the error-only the original design optics are required. This simplifies the estimation of (for example) quadrupole errors, which modify the optics all around the ring. The second advantage is that errors can be located just by looking for changes in action and phase.

In order to apply the action and phase method, it is necessary to be able to measure action and phase just before and just after the error. This is done using at least two BPMs on each side of the error under the assumption that no error exists between the two BPMs. This is not generally the case, but if the effect of the error to be measured is much larger than the effect of any error between the BPMs, the action and phase jump method usually gives a reasonably precise measurement. This is exactly the situation at the interaction regions (IRs) of a collider, because errors in the arcs usually have much smaller effects than errors in the IRs. Hence, the BPMs in the arcs can be used to estimate actions and phases before and after the IRs.

In this paper, the basic principles of the action and phase jump method are first explained (Secs. II and III), and then the simulated performance of the method is tested (Sec. IV). Finally, experimental measurements from RHIC and the SPS are presented (Secs. V, VI, and VII).

\section{THE ACTION AND PHASE JUMP METHOD}

Assume that a magnetic kick error $\theta_{z}$ (where $z$ stands for either the $x$ plane or the $y$ plane) is introduced at a longi- 
tudinal position $s=s_{\theta}$ in an otherwise perfect accelerator lattice. As a consequence, the lattice will be divided in three different regions: $s<s_{\theta}, s=s_{\theta}$, and $s>s_{\theta}$. Three independent formulas, one for every region, exactly describe the linear betatron motion of any particle, as follows.

For $s<s_{\theta}$, the trajectory is simply given by the wellknown formula,

$$
z(s)=\sqrt{2 J_{0} \beta_{z}(s)} \sin \left[\psi_{z}(s)-\delta_{0}\right],
$$

where $J_{0}$ and $\delta_{0}$ are the action and phase constants for $s<$ $s_{\theta}$, while $\beta_{z}$ and $\psi_{z}$ are the optical lattice functions. The phase space coordinate orthogonal to $z(s)$ is

$$
z^{\prime}(s)=\frac{d z}{d s}=\sqrt{2 J_{0} \gamma_{z}(s)} \cos \left[\psi_{z}(s)-\delta_{0}+\eta_{z}(s)\right],
$$

where $\eta_{z}(s)=\arctan \left[\alpha_{z}(s)\right]$ while $\alpha_{z}$ and $\gamma_{z}$ are the Courant-Snyder parameters.

For $s=s_{\theta}$, the phase space coordinates are

$$
z\left(s_{\theta}\right)=\sqrt{2 J_{0} \beta_{z}\left(s_{\theta}\right)} \sin \left[\psi_{z}\left(s_{\theta}\right)-\delta_{0}\right]
$$

and

$$
z^{\prime}\left(s_{\theta}\right)=\sqrt{2 J_{0} \gamma_{z}\left(s_{\theta}\right)} \cos \left[\psi_{z}\left(s_{\theta}\right)-\delta_{0}+\eta_{z}\left(s_{\theta}\right)\right]+\theta_{z} .
$$

These are almost like Eqs. (1) and (2) except for the last term of $z^{\prime}\left(s_{\theta}\right)$, which represents the change of the trajectory slope due to the magnetic error.

For $s>s_{\theta}$ the phase space coordinates are calculated as follows:

$$
\left(\begin{array}{c}
z(s) \\
z^{\prime}(s)
\end{array}\right)=M\left(s_{\theta} \rightarrow s\right)\left(\begin{array}{c}
z\left(s_{\theta}\right) \\
z^{\prime}\left(s_{\theta}\right)
\end{array}\right)
$$

where $M\left(s_{\theta} \rightarrow s\right)$ is the transformation matrix from $s_{\theta}$ to $s$ and is given by [13]

$$
M=\left(\begin{array}{cc}
a \cos \Delta \psi_{z}+b \sin \Delta \psi_{z} & c \sin \Delta \psi_{z} \\
d \sin \Delta \psi_{z}+e \cos \Delta \psi_{z} & f \cos \Delta \psi_{z}+g \sin \Delta \psi_{z}
\end{array}\right),
$$

where

$$
\begin{gathered}
a=\sqrt{\frac{\beta_{z}(s)}{\beta_{z}\left(s_{\theta}\right)}}, \quad b=\sqrt{\frac{\beta_{z}(s)}{\beta_{z}\left(s_{\theta}\right)}} \alpha_{z}\left(s_{\theta}\right), \\
c=\sqrt{\beta_{z}(s) \beta_{z}\left(s_{\theta}\right)}, \quad d=-\frac{1+\alpha_{z}(s) \alpha_{z}\left(s_{\theta}\right)}{\sqrt{\beta_{z}(s) \beta_{z}\left(s_{\theta}\right)}}, \\
e=\frac{\alpha_{z}\left(s_{\theta}\right)-\alpha_{z}(s)}{\sqrt{\beta_{z}(s) \beta_{z}\left(s_{\theta}\right)}}, \quad f=\sqrt{\frac{\beta_{z}\left(s_{\theta}\right)}{\beta_{z}(s)}}, \\
g=-\sqrt{\frac{\beta_{z}\left(s_{\theta}\right)}{\beta_{z}(s)}} \alpha_{z}(s),
\end{gathered}
$$

and

$$
\Delta \psi_{z}=\psi_{z}(s)-\psi_{z}\left(s_{\theta}\right) .
$$

Substituting Eqs. (3), (4), and (6), in Eq. (5), we obtain

$$
\begin{aligned}
z(s)= & \sqrt{2 J_{0} \beta_{z}(s)} \sin \left[\psi_{z}(s)-\delta_{0}\right] \\
& +\theta_{z} \sqrt{\beta_{z}(s) \beta_{z}\left(s_{\theta}\right)} \sin \left[\psi_{z}(s)-\psi_{z}\left(s_{\theta}\right)\right] .
\end{aligned}
$$

The right-hand side of Eq. (7) can also be expressed as a sinusoidal function,

$$
z(s)=\sqrt{2 J_{1} \beta_{z}(s)} \sin \left[\psi_{z}(s)-\delta_{1}\right],
$$

where $J_{1}$ and $\delta_{1}$ are the action and phase constants for $s>$ $s_{\theta}$, given by

$$
J_{1}=J_{0}+\frac{\theta_{z}^{2} \beta_{z}\left(s_{\theta}\right)}{2}+\theta_{z} \sqrt{2 J_{0} \beta_{z}\left(s_{\theta}\right)} \cos \left[\psi_{z}\left(s_{\theta}\right)-\delta_{0}\right]
$$

and

$$
\tan \delta_{1}=\frac{\theta_{z} \sqrt{\beta_{z}\left(s_{\theta}\right)} \sin \left[\psi_{z}\left(s_{\theta}\right)\right]+2 J_{0} \sin \delta_{0}}{\theta_{z} \sqrt{\beta_{z}\left(s_{\theta}\right)} \cos \left[\psi_{z}\left(s_{\theta}\right)\right]+2 J_{0} \cos \delta_{0}} .
$$

Thus the particle trajectory for $s<s_{\theta}$ [Eq. (1)] and for $s>$ $s_{\theta}$ [Eq. (8)] are described by the same functional form, but with different action and phase constants. In other words, a jump of action and phase occurs at $s=s_{\theta}$, the error location. Therefore, a plot of action and phase as a function of the axial coordinate $s$ reveals error source locations. The previous reasoning can be generalized for more than one error. In particular, it can be shown that Eq. (7) can be written as

$$
\begin{aligned}
z(s)= & \sqrt{2 J_{0} \beta_{z}(s)} \sin \left[\psi_{z}(s)-\delta_{0}\right] \\
& +\sum_{i} \theta_{z i} \sqrt{\beta_{z}(s) \beta_{z}\left(s_{\theta i}\right)} \sin \left[\psi_{z}(s)-\psi_{z}\left(s_{\theta i}\right)\right],
\end{aligned}
$$

where $\theta_{z i}$ represent any arbitrary magnetic kick error located at $s=s_{\theta_{i}}$ in an optical lattice.

The individual values of action and phase as a function of $s$ are experimentally determined from BPM measurements. Consider two adjacent BPM measurements,

$$
\begin{aligned}
z_{i} & =\sqrt{2 J_{i+1} \beta_{z i}} \sin \left(\psi_{z i}-\delta_{i+1}\right), \\
z_{i+1} & =\sqrt{2 J_{i+1} \beta_{z i+1}} \sin \left(\psi_{z i+1}-\delta_{i+1}\right),
\end{aligned}
$$

where it is assumed that there are no magnetic errors between the two BPMs, so that $J_{i}=J_{i+1}$ and $\delta_{i}=\delta_{i+1}$. Solving Eq. (12) for the constants $J_{i+1}$ and $\delta_{i+1}$ gives

$$
\begin{aligned}
J_{i+1}= & \frac{\left(z_{i} / \beta_{z i}\right)^{2}+\left(z_{i+1} / \beta_{z i+1}\right)^{2}}{2 \sin ^{2}\left(\psi_{z i+1}-\psi_{z i}\right)} \\
& -\frac{z_{i} z_{i+1} \cos \left(\psi_{z i+1}-\psi_{z i}\right)}{\beta_{z i} \beta_{z i+1} \sin ^{2}\left(\psi_{z i+1}-\psi_{z i}\right)},
\end{aligned}
$$



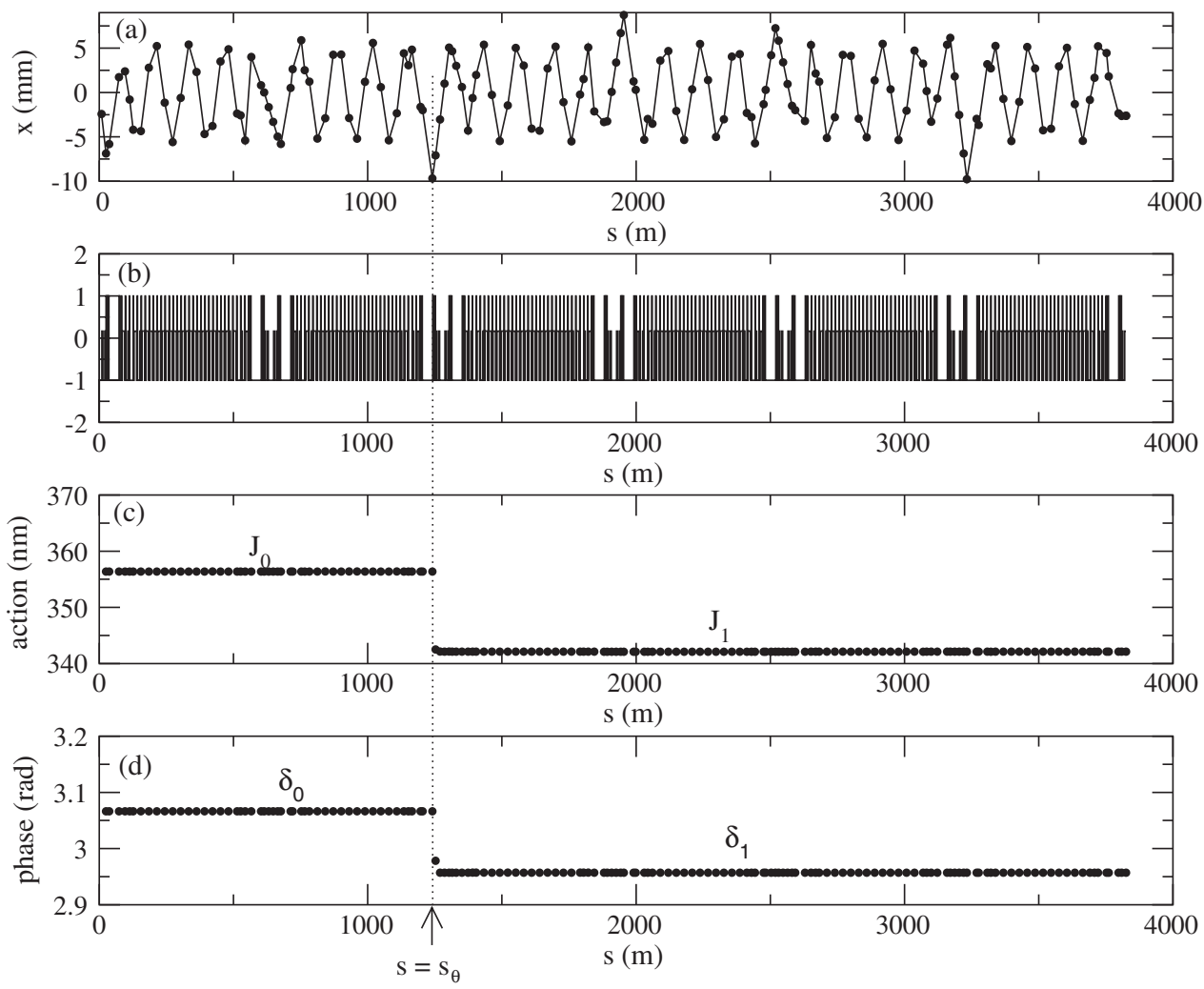

FIG. 1. (a) Simulation of a RHIC first turn trajectory with every dot representing a BPM measurement, in the presence of a magnetic kick with strength $\theta_{z}$ at $s_{\theta}=1241 \mathrm{~m}$. (b) RHIC optics with short bars representing dipoles and long bars representing quadrupoles. (c) Action analysis of the simulated first turn trajectory. (d) Phase analysis of the same trajectory.

$\tan \left(\delta_{i+1}\right)=\frac{\left(z_{i} / \beta_{z i}\right) \sin \left(\psi_{z i+1}\right)-\left(z_{i+1} / \beta_{z i+1}\right) \sin \left(\psi_{z i}\right)}{\left(z_{i} / \beta_{z i}\right) \cos \left(\psi_{z i+1}\right)-\left(z_{i+1} / \beta_{z i+1}\right) \cos \left(\psi_{z i}\right)}$

Plots of action and phase as a function of $s$ are generated by applying Eqs. (13) and (14) to all pairs of adjacent BPM readings. For example, Fig. 1(a) shows simulated BPM data representing a trajectory in RHIC with a magnetic kick error at $s_{\theta}=1241 \mathrm{~m}$. The action and phase plots in Figs. 1(c) and 1(d) are obtained by direct application of Eqs. (13) and (14). Figure 1 shows a jump in action and phase across $s_{\theta}=1241 \mathrm{~m}$, as expected.

The strength of the error can also be determined from the actions and phases measured before and after the error. Using Eqs. (7) and (8),

$$
\theta_{z}=\sqrt{\frac{2 J_{0}+2 J_{1}-4 * \sqrt{J_{0} J_{1}} \cos \left(\delta_{1}-\delta_{0}\right)}{\beta_{z}\left(s_{\theta}\right)}}
$$

where $\beta_{z}\left(s_{\theta}\right)$ is the design value of the beta function at the error.

\section{MULTIPOLE COMPONENTS OF A MAGNETIC KICK}

A magnetic kick error can have contributions from any magnetic field component-dipole, quadrupole, skew quadrupole, sextupole, et cetera. In general, the horizontal kick $\theta_{x}$ caused by a vertical deviation $\Delta B_{y}$ in a magnet of length $l$ is given by

$$
\theta_{x}=-\frac{e \Delta B_{y} l}{p}
$$

with $e$ is the electron charge and $p$ is the nominal momentum of the beam. Similarly, the vertical kick $\theta_{y}$ is

$$
\theta_{y}=\frac{e \Delta B_{x} l}{p}
$$

After expanding the magnetic field into normal and skew components $b_{n}$ and $a_{n}$, using

$$
\Delta B_{y}+i \Delta B_{x}=B \sum_{n}\left(b_{n}+i a_{n}\right)(x+i y)^{n},
$$

then Eqs. (16) and (17) lead to

$$
\begin{aligned}
\theta_{x}= & B_{0}-B_{1} x\left(s_{\theta}\right)+A_{1} y\left(s_{\theta}\right)+2 A_{2} x\left(s_{\theta}\right) y\left(s_{\theta}\right) \\
& +B_{2}\left[-x^{2}\left(s_{\theta}\right)+y^{2}\left(s_{\theta}\right)\right]+\cdots \\
\theta_{y}= & A_{0}+A_{1} x\left(s_{\theta}\right)+B_{1} y\left(s_{\theta}\right)+2 B_{2} x\left(s_{\theta}\right) y\left(s_{\theta}\right) \\
& +A_{2}\left[x^{2}\left(s_{\theta}\right)-y^{2}\left(s_{\theta}\right)\right]+\cdots,
\end{aligned}
$$

where $A_{n}=e B l a_{n} / p$ and $B_{n}=e B l b_{n} / p$. These new 
quantities completely characterize a magnetic error. The main goal of the action and phase jump method is to measure their values.

If $\theta_{x}=B_{0}$ (a dipole kick), it is clear from Eqs. (15) and (19) that it is only necessary to measure one trajectory in order to estimate the strength of the error. This remains true for any higher order component, as long as it is known to be the only component present. For example, if only quadrupole components are considered, Eqs. (19) and (20) lead to

$$
\begin{aligned}
& A_{1}=\frac{\theta_{x} y\left(s_{\theta}\right)+\theta_{y} x\left(s_{\theta}\right)}{x^{2}\left(s_{\theta}\right)+y^{2}\left(s_{\theta}\right)} \\
& B_{1}=\frac{\theta_{y} y\left(s_{\theta}\right)-\theta_{x} x\left(s_{\theta}\right)}{x^{2}\left(s_{\theta}\right)+y^{2}\left(s_{\theta}\right)},
\end{aligned}
$$

where all the quantities used to estimate $A_{1}$ and $B_{1}$ can be obtained from a single trajectory.

Several trajectories with different amplitudes must be used when several multipole components are present or suspected, and used to generate three curves: $\theta_{x}$ versus $x\left(s_{\theta}\right), \theta_{y}$ versus $x\left(s_{\theta}\right)$, and $y\left(s_{\theta}\right)$ versus $x\left(s_{\theta}\right)$, as illustrated in Fig. 2. Such curves are typically represented by

$$
\begin{aligned}
& \theta_{x}=C_{1 x} x\left(s_{\theta}\right)+C_{2 x} x^{2}\left(s_{\theta}\right)+\cdots \\
& \theta_{y}=C_{1 y} x\left(s_{\theta}\right)+C_{2 y} x^{2}\left(s_{\theta}\right)+\cdots,
\end{aligned}
$$

and

$$
y\left(s_{\theta}\right)=m x\left(s_{\theta}\right)+b .
$$

Polynomial fits to the three curves yields measured values for $C_{1 x}, C_{2 x}, C_{1 y}, C_{2 y}, m$, and $b$. Substituting Eq. (23) in Eqs. (19) and (20) shows that the relations between the

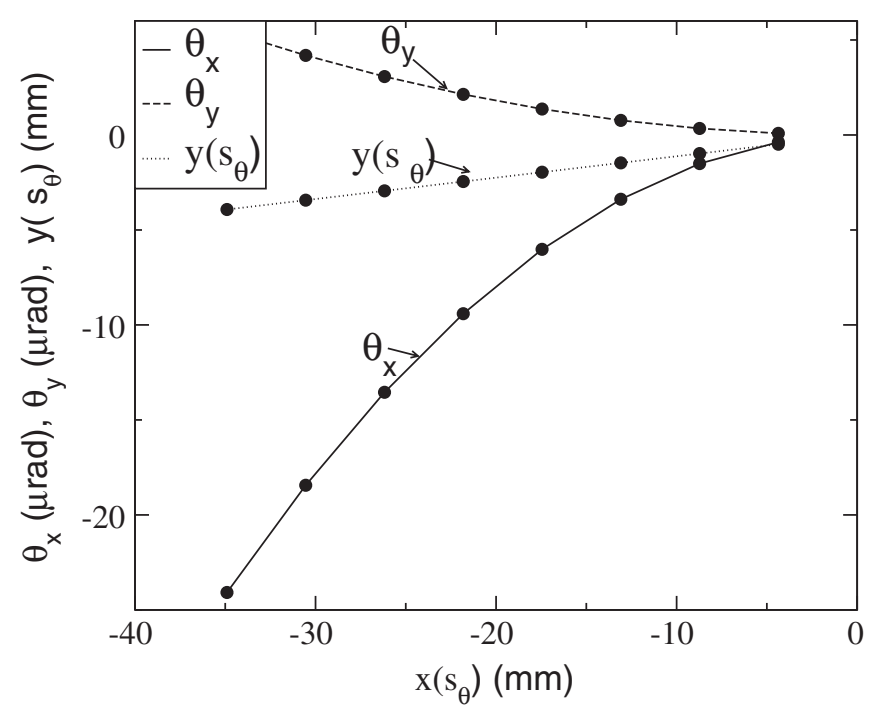

FIG. 2. Horizontal magnetic kick error $\theta_{x}$ and vertical magnetic kick error $\theta_{y}$ as functions of the horizontal particle position $x\left(s_{\theta}\right)$. The typical dependence between the horizontal particle position $x\left(s_{\theta}\right)$ and the vertical particle position $y\left(s_{\theta}\right)$ is also shown. Eight simulated first turn trajectories with different amplitudes have been used to obtain this plot. multipole components and the fitted coefficients,

$$
\begin{aligned}
& C_{1 x}=A_{1} m-B_{1}+2 A_{2} b+2 m b B_{2}, \\
& C_{1 y}=A_{1}+2 b B_{2}-2 A_{2} b m+B_{1} m, \\
& C_{2 x}=2 A_{2}-B_{2}+m^{2} B_{2}, \\
& C_{2 y}=A_{2}+2 B_{2} m-A_{2} m^{2},
\end{aligned}
$$

are a $4 \times 4$ system of the linear equation system in the unknown variables $A_{1}, A_{2}, B_{1}$, and $B_{2}$. Their solution is

$$
\begin{aligned}
& A_{1}=\frac{C_{1 x} m+C_{1 y}}{1+m^{2}}, \\
& B_{1}=-\frac{C_{1 x}-C_{1 y} m}{1+m^{2}}, \\
& A_{2}=-\frac{-C_{2 y}-2 C_{2 x} m+C_{2 y} m^{2}}{1+2 m^{2}+m^{4}}, \\
& B_{2}=-\frac{C_{2 x}-2 C_{2 y} m+C_{2 x} m^{2}}{1+2 m^{2}+m^{4}},
\end{aligned}
$$

where the coefficients $C_{2 x}$ and $C_{2 y}$ have been neglected in the calculation of $A_{1}$ and $B_{1}$, since their effect is usually much smaller than the linear terms.

Only quadrupole and sextupole components are considered in Eq. (22), because they are the most common components in practice. Dipole components are also very important, but they are readily easily suppressed from the experimental data, as discussed in Sec. V.

\section{SIMULATIONS}

The action and phase jump method is easily tested using simulated RHIC first turn trajectories with gradient errors, skew quadrupole errors, and sextupole errors. Simulated trajectories are generated with MAD [14], which is able to simulate both single turn trajectories and also closed orbits. Errors are simulated by setting to a certain strength a quadrupole, a skew quadrupole, or a sextupole at $s_{\theta}=$ $1241 \mathrm{~m}$. The simulated trajectories are then analyzed, and the measured error is compared with set error. The difference $\epsilon$ is the error of the method.

\section{A. Gradient error}

An integrated gradient error of $B_{1}=1 \times 10^{-3} \mathrm{~m}^{-1}$ is added, and four trajectories with different amplitudes are simulated leading to four plots of trajectories, actions, and phases like the one shown in Fig. 1. Those plots along with Eq. (15) finally lead to Fig. 3. The simulated trajectories contain only a gradient error $\left(A_{1}=B_{2}=A_{2}=0\right)$, with no coupling ( $m=0)$, so Eqs. (22a) and (24a) lead to

$$
\theta_{x}=-B_{1} x\left(s_{\theta}\right) .
$$

The slope measured from the data shown in Fig. 3 is very close to the expected value of $-B_{1}=-1 \times 10^{-3}$.

The method error $\epsilon$ was calculated using the same procedure for ten different gradient errors, as shown in Fig. 4. The largest value of $\epsilon$ in Fig. 4, for typical values of 


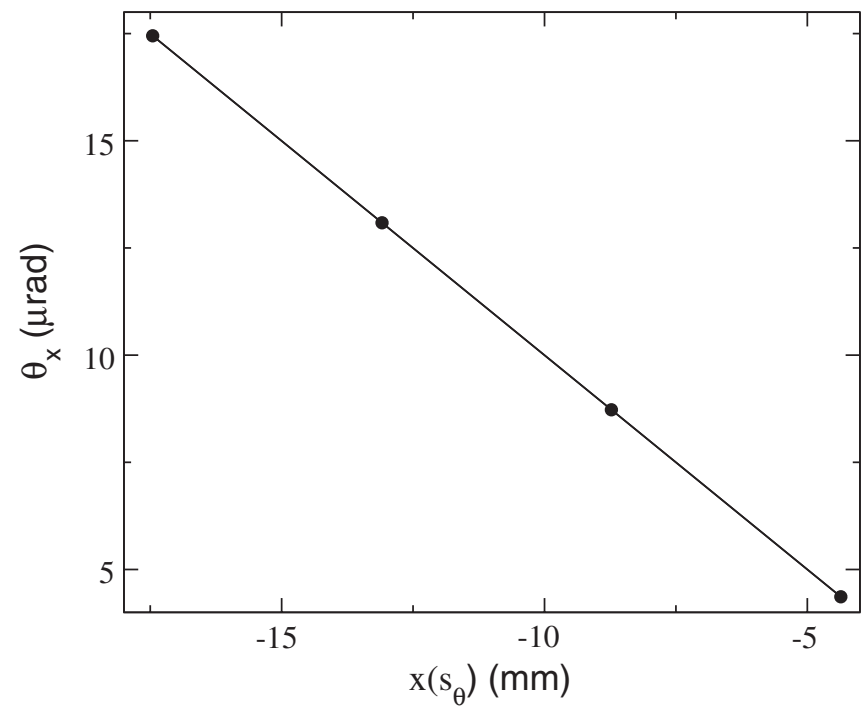

FIG. 3. The measured horizontal quadrupole kick error $\theta_{x}$ as a function of the horizontal position of the particle $x\left(s_{\theta}\right)$, in response to a gradient error of $1 \times 10^{-3} \mathrm{~m}^{-1}$ intentionally introduced in the lattice at $s_{\theta}=1241 \mathrm{~m}$. The slope of the curve, $-0.99969 \times 10^{-3} \mathrm{~m}^{-1}$, is very close to the expected value.

gradient errors at RHIC, suggests that the method could be accurate to within a $0.04 \%$ uncertainty.

\section{B. Skew quadrupole error}

A similar procedure can be followed for skew quadrupole errors. However, in this case the horizontal trajectory is perturbed by a vertical magnetic kick, so that the new version of Fig. 3 plots $\theta_{y}$ versus $x\left(s_{\theta}\right)$ instead of $\theta_{x}$ versus $x\left(s_{\theta}\right)$. Again, ten different skew quadrupole strength settings are simulated and their corresponding $\epsilon$ values are calculated. Figure 5 shows that $\epsilon$ oscillates around $0.01 \%$.

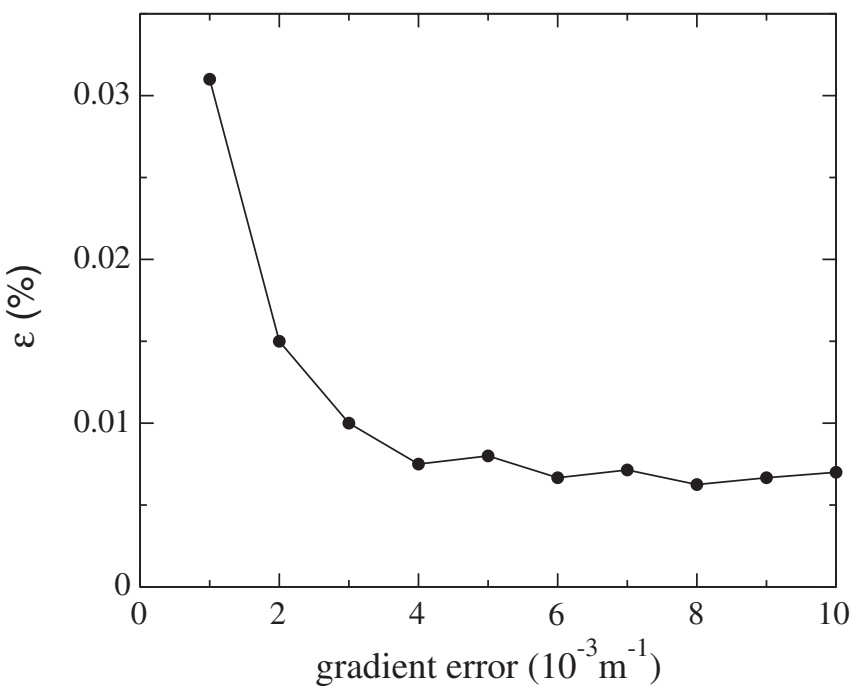

FIG. 4. The error of the method $\epsilon$ as a function of the gradient error strength, predicting the method error to be less than $0.04 \%$ for RHIC gradient errors larger than $10^{-3} \mathrm{~m}^{-1}$.

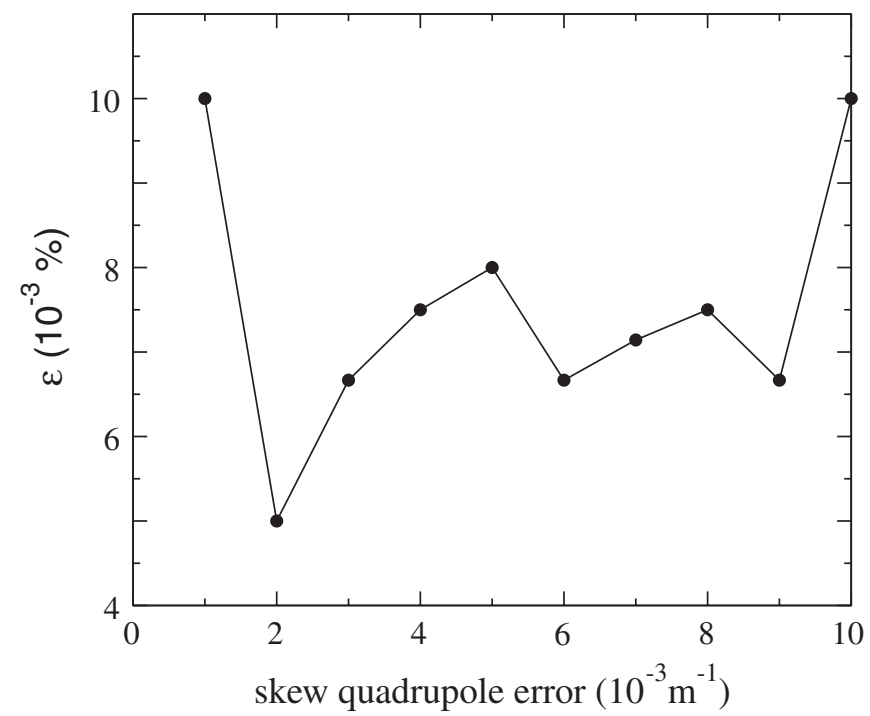

FIG. 5. Simulations predict that the action and phase jump method determines skew quadrupole errors within an uncertainty as small as $0.01 \%$.

\section{Sextupole error}

Sextupole errors lead to a nonlinear dependence of $\theta_{x}$ on $x\left(x_{\theta}\right)$, as shown in Fig. 6, because when only a sextupole error $B_{2}$ is present the horizontal kick is

$$
\theta_{x}=-B_{2} x^{2}\left(s_{\theta}\right)
$$

A quadratic fit of the data in Fig. 6 gives the value of $B_{2}$. Sextupoles are included in the simulation through the integrated sextupole strength K2L (MAD notation), which is related to the magnetic fields by

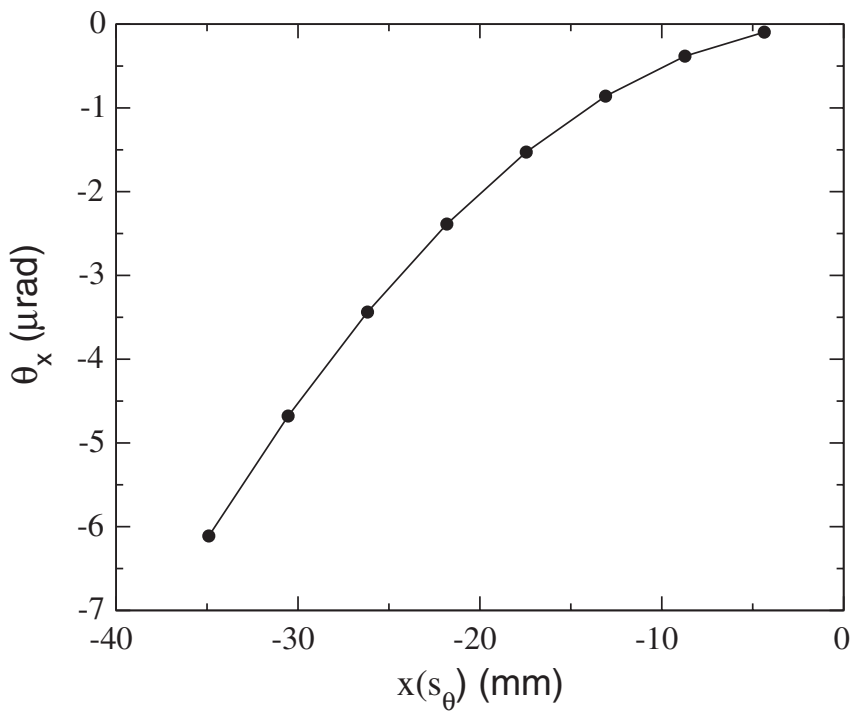

FIG. 6. The horizontal kick error $\theta_{x}$ is a nonlinear function of the trajectory excursion $x\left(s_{\theta}\right)$ when a sextupole error is present. A quadratic fit of the plot gives the sextupole strength. 


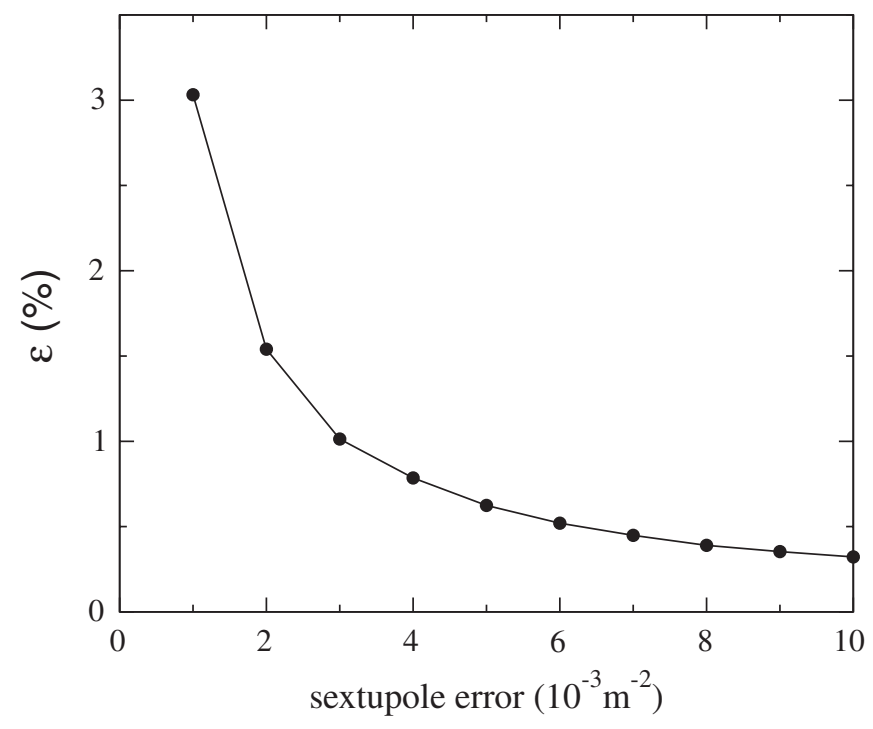

FIG. 7. The action and phase jump method determines sextupole errors within a $3 \%$ uncertainty in the range of strengths of interest for RHIC sextupoles.

$$
K 2 L=\frac{B^{\prime \prime} l}{B_{\rho}} .
$$

On the other hand,

$$
B_{2}=\frac{e B l b_{2}}{p}=\frac{B l b_{2}}{B_{\rho}}=\frac{B^{\prime \prime} l}{2 B_{\rho}}
$$

and hence

$$
K 2 L=2 B_{2} .
$$

Thus, the quadratic coefficient obtained from fitting the $\theta_{x}$ versus $x\left(s_{\theta}\right)$ data must be multiplied by two in order to recover the sextupole strength values set in the MAD files.

The differences between the sextupole error settings and the values recovered using the action and phase jump method are significantly larger than in the two previous cases, as shown in Fig. 7. Nonetheless, these differences are smaller than $3 \%$ in the range of interest for RHIC sextupoles. The differences decrease quite rapidly as the sextupole strength increases.

\section{LINEAR EXPERIMENTS WITH BEAM IN RHIC}

It is necessary to excite large amplitude trajectories in order to experimentally test the action and phase jump method in an accelerator. In RHIC, this is easily done by turning on any of the dipole correctors installed throughout the accelerator and recording the corresponding trajectories as turn by turn data.
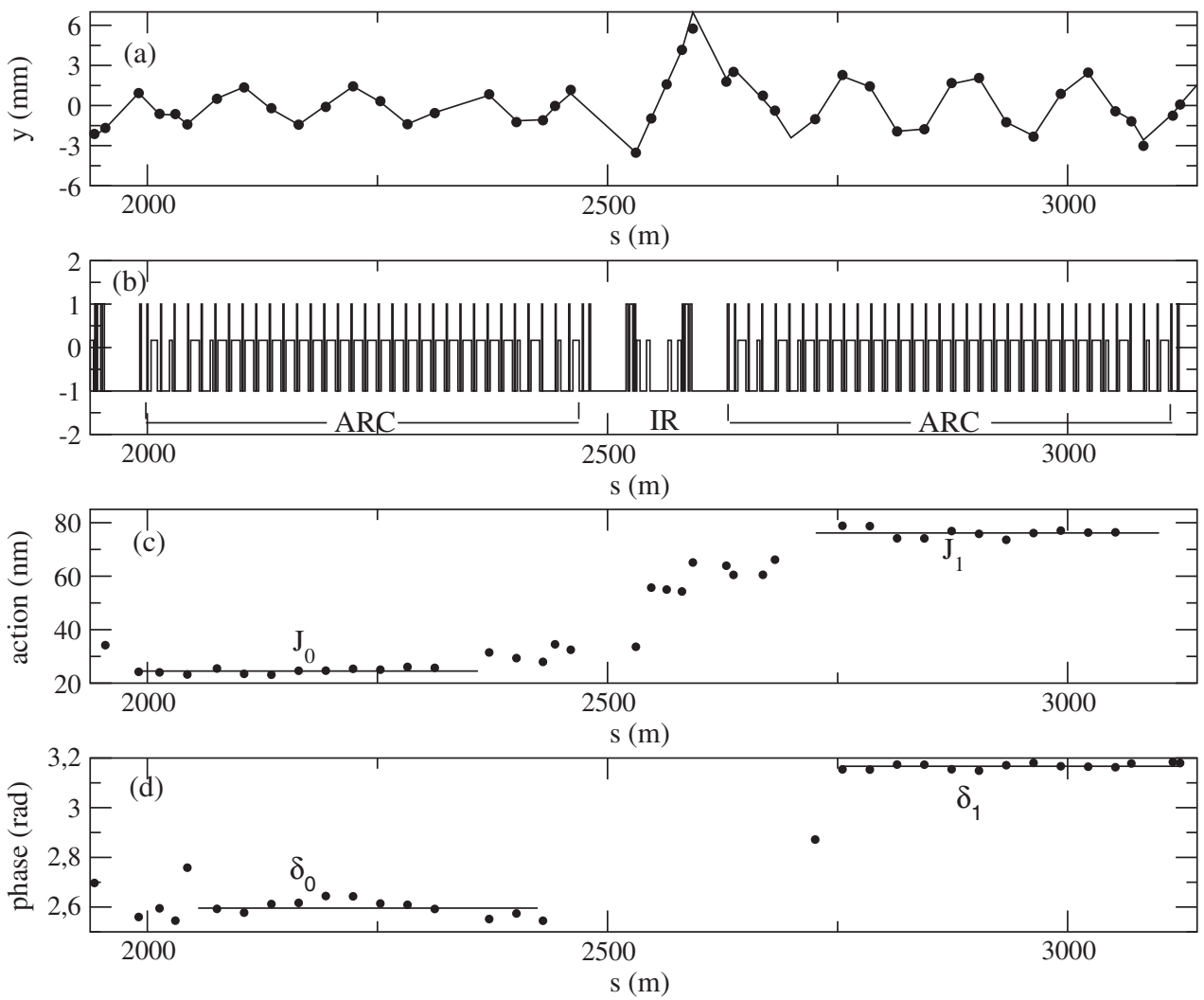

FIG. 8. (a) One of the first difference orbits obtained from beam in RHIC in the year 2000. Each dot represents a BPM measurement. (b) Lattice representation of RHIC. (c) Action plot obtained by applying Eq. (13) to all pairs of adjacent BPM measurements in the ring. (d) Phase plot obtained by applying Eq. (14) to all pairs of adjacent BPM measurements in the ring. 
It is necessary to subtract the baseline orbit (which exists in the accelerator before turning on any dipole corrector) from these turn by turn trajectories, in order to generate "difference orbits" in which dipole errors and BPM systematic errors are both suppressed. For the analysis presented in this section, the difference orbits were constructed with the first turn of the baseline and the first turn of the large amplitude trajectory.

Equations (13) and (14) are applied to every pair of adjacent BPMs in every difference orbit, to obtain plots like that shown in Fig. 8. In this figure the action and phase plots of RHIC difference orbits are approximately constant along the arcs, but big jumps are visible at the IRs. Such behavior is expected because the size of the action jump inferred from Eq. (9) is determined by the product of $\theta_{z}^{2} \beta_{z}\left(s_{\theta}\right)$, and the beta functions in the interaction regions are much larger than in the arcs. Even small IR errors lead to larger effects than are typical from arc errors.

\section{A. Data from the RHIC 2002 run}

The action and phase method not only points to error locations, but also estimates error strengths.

These capabilities were tested with beam during the RHIC 2002 proton run at injection, by introducing six different known skew quadrupole strengths at the bi8-qs3 corrector in the 8 o'clock IR. Large beam trajectories were excited, one for every bi8-qs3 setting, with one of the RHIC dipole correctors and recorded as turn by turn data. The dipole corrector was chosen at a location such that the orbit excursion at bi8-qs3 was near a maximum. From the turn by turn data, only the first turn in each case was used to build the difference orbit as explained above.

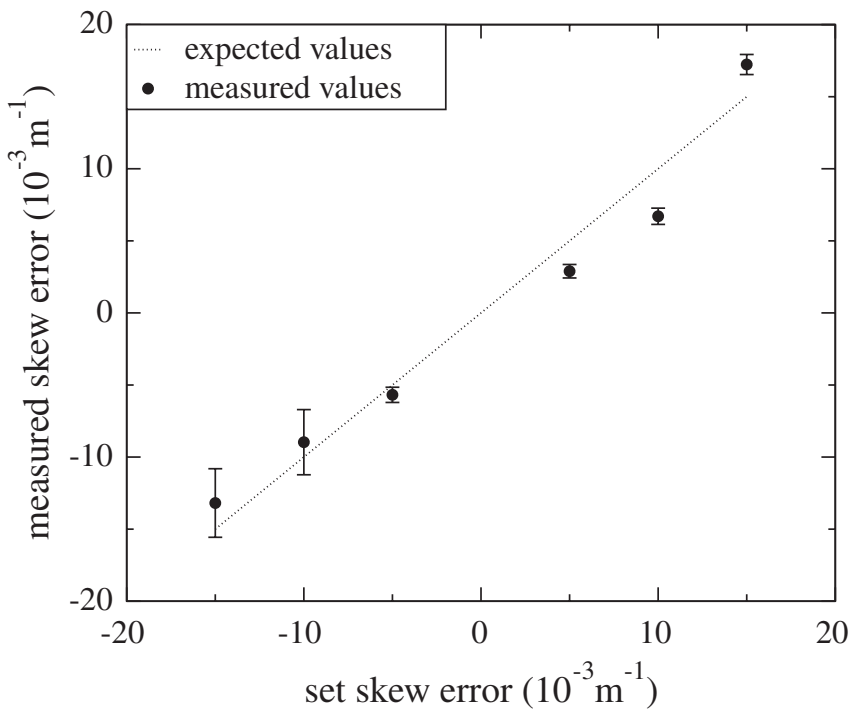

FIG. 9. Relation between the skew quadrupole errors intentionally introduced in the accelerator and the measured errors obtained from action and phase analysis of RHIC difference orbits.
Actions $J_{0}$ and $J_{1}$ and phases $\delta_{0}$ and $\delta_{1}$ were estimated around IR8 using data sets similar to that shown in Fig. 8. These estimations were obtained by averaging the individual actions and phases in the arcs around IR8. Actions and phases that deviated more than 3 standard deviations from the average were discarded. Equations (15) and (21a) were used to obtain the measured error values shown in Fig. 9, once the actions and phases before and after the error were known.

Since six first turn orbits were available for every setting of the skew quadrupole and six baselines, it was possible to build 36 difference orbits. Therefore, six different values for every skew quadrupole setting were obtained with Eq. (21a). Three times the standard deviation of those values gives the height of the error bar at each point of Fig. 9. Most of the measured skew quadrupole values were close to their set values - a linear regression of the experimental points in Fig. 9 gave a slope of $1.00 \pm 0.12$, agreeing well with the expected value 1.0 , but with an uncertainty of $12 \%$.

\section{B. Beam position monitor gain errors}

BPM gain errors are often a point of concern when analyzing experimental data to extract optical parameters of a lattice. To study their effect on the action and phase method, first consider the case when all BPMs in the accelerator have the same error gain so that the (difference) beam position $z_{i}^{*}$ reported by any BPM is related to the real beam position $z_{i}$ by

$$
z_{i}^{*}=k z_{i}
$$

where $k$ represents the gain error. Using Eqs. (13) and (14) it can be shown that

$$
\begin{aligned}
& J_{0}^{*}=k^{2} J_{0}, \quad J_{1}^{*}=k^{2} J_{1}, \\
& \delta_{0}^{*}=\delta_{0}, \quad \delta_{1}^{*}=\delta_{1},
\end{aligned}
$$

where $J_{0}^{*}, J_{1}^{*}, \delta_{0}^{*}$, and $\delta_{1}^{*}$ are the actions and phases calculated with the BPM measurements $z_{i}^{*}$. Replacing Eq. (32) in Eq. (15), the estimated strength error $\theta_{z}^{*}$ becomes

$$
\theta_{z}^{*}=k \theta_{z}
$$

When Eqs. (31) and (33) are replaced in Eqs. (19) and (20), we obtain

$$
\begin{aligned}
\theta_{x}^{*}= & B_{0}^{*}-B_{1}^{*} x^{*}\left(s_{\theta}\right)+A_{1}^{*} y^{*}\left(s_{\theta}\right)+2 A_{2}^{*} x^{*}\left(s_{\theta}\right) y^{*}\left(s_{\theta}\right) \\
& +B_{2}^{*}\left[-x^{* 2}\left(s_{\theta}\right)+y^{* 2}\left(s_{\theta}\right)\right]+\cdots, \\
\theta_{y}^{*}= & A_{0}^{*}+A_{1}^{*} x^{*}\left(s_{\theta}\right)+B_{1}^{*} y^{*}\left(s_{\theta}\right)+2 B_{2}^{*} x^{*}\left(s_{\theta}\right) y^{*}\left(s_{\theta}\right) \\
& +A_{2}^{*}\left[x^{* 2}\left(s_{\theta}\right)-y^{* 2}\left(s_{\theta}\right)\right]+\cdots,
\end{aligned}
$$

where 


$$
\begin{aligned}
& B_{0}^{*}=k B_{0}, \\
& A_{1}^{*}=A_{1}, \quad B_{1}^{*}=B_{1}, \\
& A_{2}^{*}=A_{2} / k, \quad B_{2}^{*}=B_{2} / k .
\end{aligned}
$$

Equation (35) shows that, while a systematic gain error in all BPMs does not affect the estimation of quadrupole errors, nonetheless the estimations of dipole errors and sextupole errors are affected. This apparent impasse is solved with a simple measurement: a dipole corrector in the accelerator is set to a strong and known value $B_{0}$. After applying action and phase analysis to the resultant orbit, $B_{0}^{*}$ and therefore $k$ are found using Eq. (35a).

It is possible to have acceptable estimations of $J_{0}, J_{1}, \delta_{0}$, and $\delta_{1}$ even when random BPMs gain errors are present, if several measurements are available. For example, in the RHIC case it is reasonable to consider the actions and phases within an arc to be constant, yielding ten measurements per arc that can be averaged. The fluctuations of $J_{0}$, $J_{1}, \delta_{0}$, and $\delta_{1}$ around their arc averages that are visible in Fig. 8 are significantly smaller than the jumps in the IRs. These fluctuations may be due either to magnetic errors within the arcs or to gain errors-they are an upper bound on the random BPM gain errors.

\section{NONLINEAR EXPERIMENTS WITH BEAM IN THE SPS}

Nonlinear experiments with sextupoles were conducted during the 2003 RHIC deuterium-gold run, by powering a sextupole corrector at the 8 o'clock interaction region. A set of orbits with different amplitudes was taken while the sextupole strength was kept constant, yielding data similar to that shown in Fig. 6. Unfortunately, the results of these experiments were inconclusive. Even though the trend of the measurements was as expected, the measurement scatter was significant when compared with the expected values and the error bars $[15,16]$. Fortunately, experimental verification of the ability of the action and phase jump method to resolve sextupole components was possible thanks to SPS turn by turn beam data that was originally taken during sextupole resonance driving term studies [17].

During those studies, a beam with an energy of $80 \mathrm{GeV}$ (injection energy for SPS is $26 \mathrm{GeV}$ ) was excited with a $Q$ kicker (a kicker used to measure transversal tunes) to produce turn by turn trajectories of seven different amplitudes while eight strong sextupoles were activated around the ring. At least three turn by turn trajectories were recorded for every strength of the kicker which makes 21 turn by turn trajectories available for the studies presented in this section. Turn by turn difference orbits were built subtracting the orbit before the kick from the orbits just after the kick. Phase analysis of the turn by turn difference orbits revealed jumps at the strong sextupole locations, as shown, for example, in Fig. 10, where the jumps appear large in only some turns, because the protons follow different trajectories on each turn. The turn with the largest

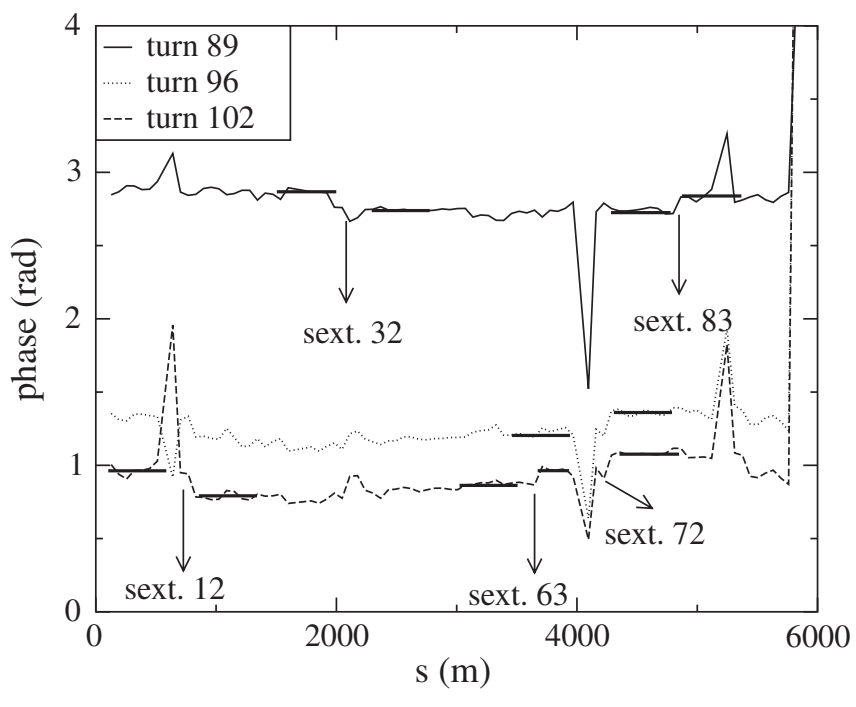

FIG. 10. Phase analysis of a SPS turn by turn difference orbit. The sextupoles that were intentionally introduced in the accelerator can be clearly identified by the jumps in phase (taken from Ref. [17]).

jumps in action and phase (optimal turn) gave the most accurate estimates for a particular error.

Using the optimal turns of the 21 turn by turn difference orbits and following the procedure explained in Sec. IV C around sextupole 63 (at $s=3646 \mathrm{~m}$ ), Fig. 11 is obtained. From this figure, the strength of sextupole 63 was estimated to be $B_{2}=(0.219 \pm 0.016) \mathrm{m}^{-2}$, yielding an integrated sextupole strength of $(0.438 \pm 0.032) \mathrm{m}^{-2}$ ac-

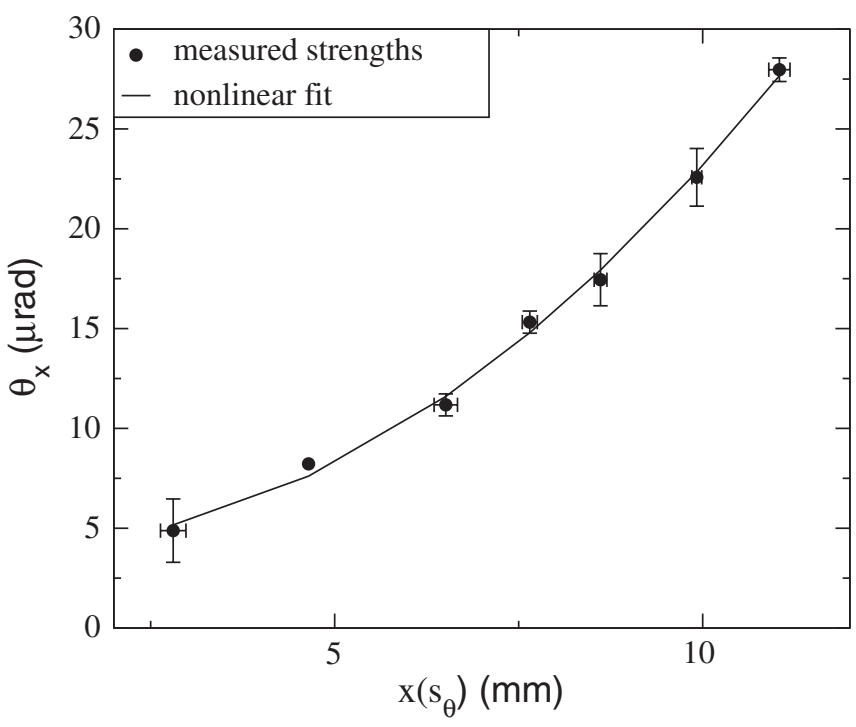

FIG. 11. Measured horizontal kick error as a function of the horizontal beam position for sextupole 63 in the SPS ring. Optimal turns of turn by turn difference orbits with seven different amplitudes were used to obtain this curve. A quadratic fit gave an integrated sextupole strength in good agreement with the actual set strength value (taken from Ref. [17]). 
cording to Eq. (30), with a relative uncertainty of about 7\%. Each point with its error bar in Fig. 11 was obtained with the three turn by turn trajectories taken for every different kicker strength. This measurement was in good agreement with the integrated strength of $0.446 \mathrm{~m}^{-2}$ that was set in sextupole 63 .

\section{DIFFERENCE ORBITS FROM A SINGLE TURN BY TURN MEASUREMENT}

Action and phase jump analysis of experimental data shown in previous sections were based on conventional difference orbits which were obtained subtracting a baseline orbit from a large amplitude beam orbit. It is also possible to build difference orbits from two large amplitude beam orbits like, for example, two turns of the same multiturn beam trajectory. In this section, we show that these new difference orbits not only help to eliminate contributions due to dipole errors as in the case of conventional difference orbits but they could also magnify the magnetic error under evaluation and significantly reduce the noise.

Let us assume a lattice with a quadrupole error $\theta_{q}=B_{1} x$ at $s=s_{\theta q}$ that wants to be estimated using action and phase analysis. Let us also assume that there is a dipole error $\theta_{d}=B_{0}$ at $s=s_{\theta d}$. According to Eq. (11), a large amplitude beam orbit in such a lattice can be represented by

$$
\begin{aligned}
x(s)= & \sqrt{2 J \beta_{x}(s)} \sin \left[\psi_{x}(s)-\delta\right] \\
& +B_{0} \sqrt{\beta_{x}(s) \beta_{x}\left(s_{\theta d}\right)} \sin \left[\psi_{x}(s)-\psi_{x}\left(s_{\theta d}\right)\right] \\
& +B_{1} x\left(s_{\theta q}\right) \sqrt{\beta_{x}(s) \beta_{x}\left(s_{\theta q}\right)} \sin \left[\psi_{x}(s)-\psi_{x}\left(s_{\theta q}\right)\right] .
\end{aligned}
$$

A baseline orbit can be represented exactly by the same equation but in this case $J$ and $x\left(s_{\theta q}\right)$ are generally much smaller than in the previous case. Hence, the corresponding (conventional) difference orbit $\Delta x(s)$ is given by

$$
\begin{aligned}
\Delta x(s)= & \sqrt{2 J \beta_{x}(s)} \sin \left[\psi_{x}(s)-\delta_{2}\right]+B_{1} x\left(s_{\theta q}\right) \\
& \times \sqrt{\beta_{x}(s) \beta_{x}\left(s_{\theta q}\right)} \sin \left[\psi_{x}(s)-\psi_{x}\left(s_{\theta q}\right)\right],
\end{aligned}
$$

where it is clear that the contribution from the dipole error has been eliminated from the orbit.

Now, let us consider two orbits $x_{2}(s)$ and $x_{1}(s)$ of large and similar amplitudes $J_{2}$ and $J_{1}$ in the same lattice. Their difference is given by

$$
\begin{aligned}
\Delta x(s)= & \sqrt{2 J_{2} \beta_{x}(s)} \sin \left[\psi_{x}(s)-\delta_{2}\right] \\
& -\sqrt{2 J_{1} \beta_{x}(s)} \sin \left[\psi_{x}(s)-\delta_{1}\right]+B_{1}\left[x_{2}\left(s_{\theta q}\right)\right. \\
& \left.-x_{1}\left(s_{\theta q}\right)\right] \sqrt{\beta_{x}(s) \beta_{x}\left(s_{\theta q}\right)} \sin \left[\psi_{x}(s)-\psi_{x}\left(s_{\theta q}\right)\right],
\end{aligned}
$$

where the first two terms can be combined into a single sine function and Eq. (39) becomes very similar to Eq. (38) and, hence, action and phase analysis can give the value of $B_{1}$. Notice also that the effect of the quadrupole error is proportional to the difference of orbit trajectory or beam positions at $s=s_{\theta q}$. If the orbits $x_{2}(s)$ and $x_{1}(s)$ are chosen such that $x_{2}\left(s_{\theta q}\right)$ and $x_{1}\left(s_{\theta q}\right)$ are maximum and opposite, the effect of the quadrupole error in this new difference orbit can be twice the effect of the same error in a conventional difference orbit.

There is still a second advantage of the new difference orbits when compared to the conventional difference orbits. Orbits used to build a new difference orbit can be any pair of turns of different turn by turn trajectories or even turns of the same turn by turn trajectory. If the turns are taken from the same trajectory, significant noise reduction can be achieved.

This was recently verified (see [18]) building first, a difference orbit in the conventional way, obtaining its corresponding action and phase plots (see Fig. 12), and
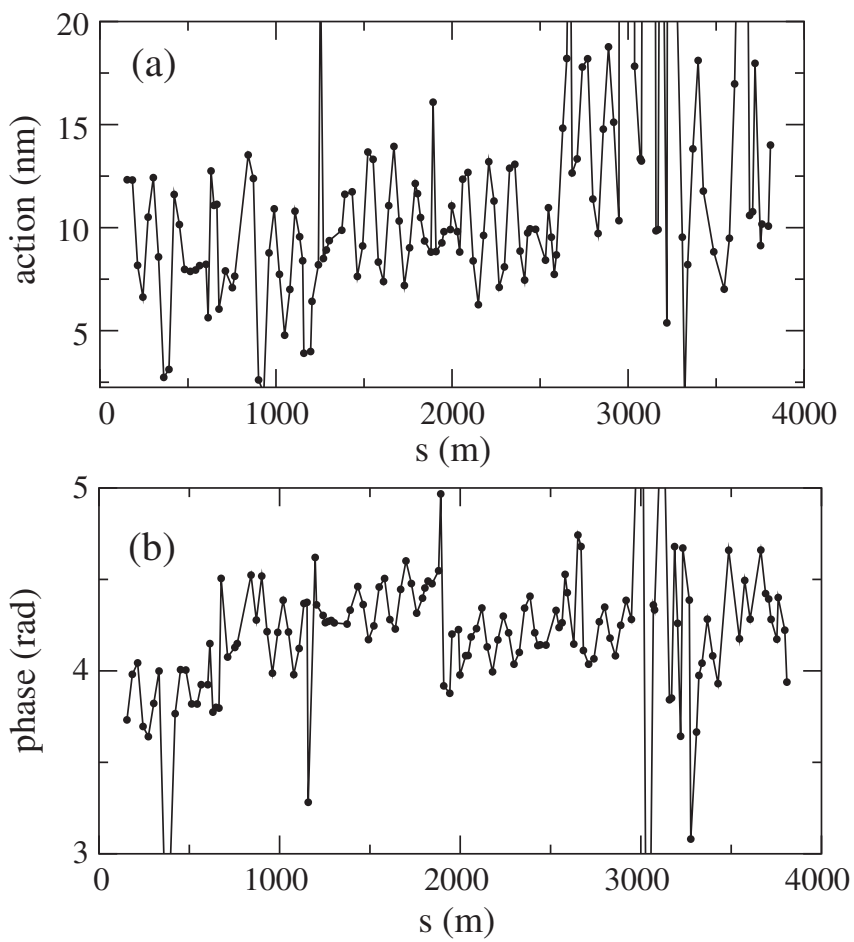

FIG. 12. Action and phase analysis of a RHIC difference orbit taken during the 2002 proton run. The difference is built out of two separated first turn orbits named bi8-qs3.-0.0010.8h.sdds and baseline.1.1.8h.sdds. 

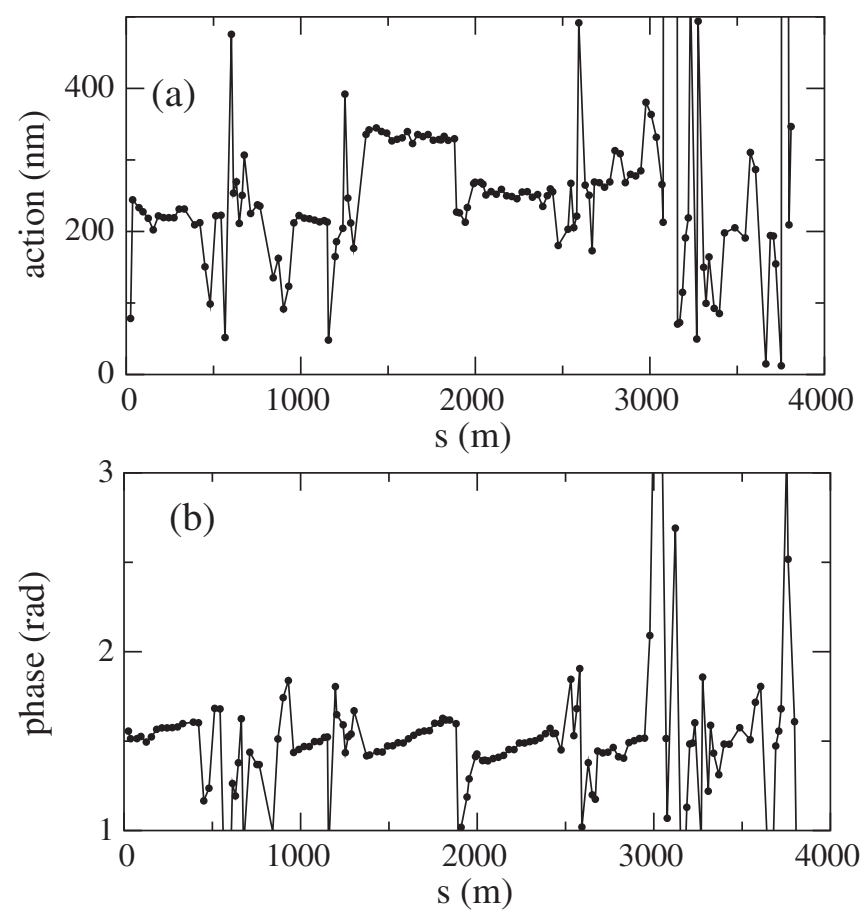

FIG. 13. Action and phase jump analysis of a RHIC difference orbit. This time the difference is built by substrating orbit in turn 17 from orbit in turn 15 of the turn by turn trajectory named bi8qs3.-0.0010.8h.sdds.

then building a difference orbit with two turns of the same turn by turn trajectory and obtaining its corresponding action and phase plots (see Fig. 13). Using the conventional difference orbits, the noise was as big as the jumps at the IRs. Using the new difference orbits, the noise in the arcs is dramatically reduced and the jumps in the IRs can be

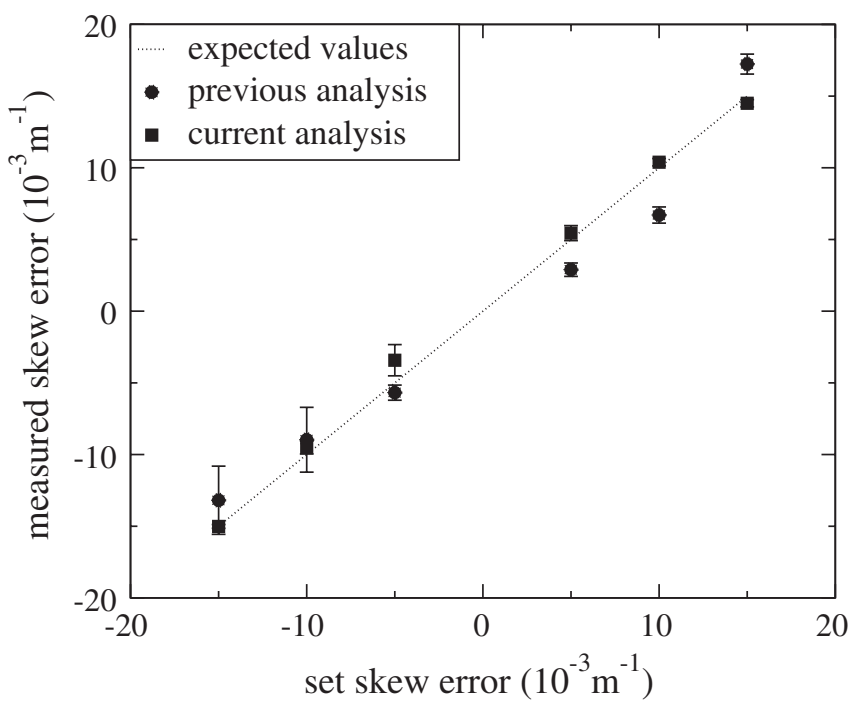

FIG. 14. Skew quadrupole errors estimated from the conventional difference orbits (circles) and skew quadrupole errors estimated from the new difference orbits (squares). This figure was taken from Ref. [18]. clearly seen. This example is an extreme case and the improvement is not always so notorious, but in general there is noise reduction when the difference orbit is built as described in the second case.

The experiment discussed in Sec. V was analyzed again, but this time the new difference orbits were used instead of the conventional difference orbits.

The results can be seen in Fig. 14 where the horizontal axis represents the skew quadrupole error set in the skew quadrupole corrector bi8-qs3, and the vertical axis represents the skew quadrupole error values estimated with action and phase jump analysis on the experimental orbits. The circles correspond to skew errors obtained with the previous analysis while the squares correspond to the new analysis. The errors bars for each point of the new analysis were obtained by building six different difference orbits from 12 turns of the same turn by turn trajectory. A linear regression of the experimental points in Fig. 14 gives a slope of $0.996 \pm 0.012$ which leads to a $1.2 \%$ uncertainty on the determination of skew quadrupole components, an improvement of 1 order of magnitude when compared to the old analysis.

\section{CONCLUSIONS}

Exact expressions were derived that relate localized errors in an accelerator lattice to the action and phase jumps that they cause.

Simulations showed that these relationships can be used to estimate small quadrupole errors within an uncertainty of $0.04 \%$ under ideal conditions. These uncertainties dropped to the $0.01 \%$ level as the quadrupole error strength increases. The estimation of sextupole errors from simulated orbits has larger uncertainties, reaching $3 \%$ for small sextupole errors and decreasing rapidly for larger errors.

Beam experiments with quadrupole errors placed at one particular location in RHIC demonstrated measurements within an average uncertainty of $12 \%$. An approach that constructed difference orbits from consecutive turns of a single multiturn measurement showed a significant improvement, reducing the uncertainty of quadrupole error measurements in RHIC from $12 \%$ to $1.2 \%$.

Beam experiments in the SPS with strong sextupoles showed that the strength of the sextupoles can be recovered within a $7.3 \%$ uncertainty.

\section{ACKNOWLEDGMENTS}

Many thanks go to Todd Satogata, who provided valuable help in the experiments, to Vadim Ptitsyn, for help and suggestions in the analysis of data, and to all RHIC staff. We are also grateful to Rogelio Tomás García, who provided critical SPS data for the sextupole analysis presented here. J. Cardona thanks DINAIN, the National Division of Research at the National University of Colombia, for financial support. 
[1] J. Safranek, Nucl. Instrum. Methods Phys. Res., Sect. A 388, 27 (1997).

[2] D. Robin, J. Safranek, G. Portmann, and H. Nishimura, in Proceedings of the 1996 European Particle Accelerator Conference, Sitges, Spain (Institute of Physics, Bristol, UK, 1996), p. 971.

[3] C. Steier, A. Wolski, S. Ecklund, J. Safranek, P. Tenenbaum, A. Terebilo, J. Turner, and G. Yocky, in Proceedings of the 2004 European Particle Accelerator Conference, Lucerne, Switzerland (EPS-IGA/CERN, Geneva, 2004), p. 1488.

[4] V. Sajaev and L. Emery, in Proceedings of the 2002 European Particle Accelerator Conference, Paris, France (EPS-IGA/CERN, Geneva, 2002), p. 742.

[5] V. Ptitsyn, J. Cardona, F. Pilat, and J. P. Koutchouk, in Proceedings of the 2001 Particle Accelerator Conference, Chicago, IL (IEEE, Piscataway, NJ, 2001), p. 3132.

[6] F. Pilat, P. Cameron, V. Ptitsyn, and J. P. Koutchouck, in Proceedings of the 2002 European Particle Accelerator Conference, Paris, France, Ref. [4], p. 1281.

[7] F. Pilat, S. Binello, P. Cameron, and V. Ptitsyn, in Proceedings of the 2003 Particle Accelerator Conference, Portland, OR (IEEE, Piscataway, NJ, 2003), p. 1703.

[8] F. Pilat, Y. Luo, N. Malitsky, and V. Ptitsyn, in Proceedings of the 2005 Particle Accelerator Conference, Knoxville, TN (IEEE, Piscataway, NJ, 2005), p. 601.
[9] J. Cardona, Ph.D. thesis, Stony Brook University, 2003.

[10] W. Fischer, F. Schmidt, and R. Tomás, in Proceedings of the 2003 Particle Accelerator Conference, Portland, OR, Ref. [7], p. 2228.

[11] R. Tomás, M. Bai, W. Fischer, A. Franchi, and G. Rumolo, in Proceedings of the 2004 European Particle Accelerator Conference, Switzerland, Ref, [3], p. 2242.

[12] R. Calaga, M. Bai, S. Peggs, T. Roser, and T. Satogata, in Proceedings of the 2004 European Particle Accelerator Conference, Switzerland, Ref, [3], p. 1541.

[13] D. Edwards and M. Syphers, An Introduction to the Physics of High Energy Accelerators (John Wiley \& Sons, New York, 1993).

[14] H. Grote and F. Iselin, The MAD Program (European Organization for Nuclear Research, Geneva, Switzerland, 1996).

[15] J. Cardona, S. Peggs, T. Satogata, and R. Tomas, in Proceedings of the 2003 Particle Accelerator Conference, Portland, OR, Ref. [7], p. 2901.

[16] J. Cardona, Ph.D. thesis, Stony Brook University, 2003.

[17] J. Cardona and R. Tomas, in Proceedings of the 2005 Particle Accelerator Conference, Knoxville, TN, Ref. [8], p. 2012.

[18] J. Cardona, in Proceedings of the 2007 Particle Accelerator Conference, Albuquerque, NM (IEEE, Piscataway, NJ, 2007), p. 3244. 